Gut, 1963, 4, 42

\title{
Studies in dogs on the biphasic nature of the gastric secretory response to hypoglycaemia and other stimuli with special reference to the role of the adrenals
}

\author{
W. SIRCUS ${ }^{1}$, C. J. W. HUSTON, R. M. PRESHAW ${ }^{2}$, \\ H. BASSÖE ${ }^{3}$, and R. A. HARKNESS \\ From the Gastrointestinal Unit, the Western General Hospital, Edinburgh, the \\ Department of Medicine, University of Edinburgh, and the \\ Clinical Endocrinology Research Unit, University of Edinburgh
}

EDITORIAL SYNOPSIS The gastric secretory response of dogs with fundal pouches to insulin hypoglycaemia is biphasic in character, the two secretory peaks being separated by some hours. The first response depends on the intact innervation of the pouch and is attributed to direct vagal action on the parietal cell. Experiments have been made to test the hypothesis that the second peak is due to activation of a pituitary adrenal mechanism.

The second phasic response does not depend on the intact innervation of the pouch and does not coincide with the period of hypoglycaemia. No relationship has been established with the second phase and plasma cortisol levels nor is this peak abolished by bilateral adrenalectomy. The second acid secretory response, however, is only evident when acid secretion of the main stomach makes prolonged contact with the antral mucosa; it is abolished by antrectomy. The hypothesis is put forward that the pattern of response following insulin hypoglycaemia after the initial vagal response is attributable to an interaction of acid-inhibiting and acid-stimulating mechanisms of gastric acid secretion mediated through the gastric antrum.

\section{The pattern of the biphasic response and the relation to gastric innervation and to hypoglycaemia}

It has been reported that electrode stimulation of the anterior hypothalamus in monkeys results in an immediate secretion of gastric juice which can be prevented by prior vagotomy. Stimulation of the posterior hypothalamus, however, is followed by gastric secretion after a delay of some hours and this delayed response is abolished by prior bilateral adrenalectomy (Porter, Movius, and French, 1953). As an explanation of the latter observations these authors postulated a pituitary-adrenal phase of gastric secretion. In an extension of the study, the injection of insulin was used to stimulate gastric

'Part time member of the external staff, Medical Research Council.

${ }^{2}$ Present address: The Department of Clinical Surgery, University of Edinburgh.

3Present address: The Department of Medicine, The Medical School, University of Bergen, Norway. secretion, and the resulting changes in the $p \mathrm{H}$ of the gastric juice were claimed to represent the summation of immediate (vagal) and delayed (pituitaryadrenal) phases of the gastric secretory response (French, Longmire, Porter, and Movius, 1953).

The present study was undertaken to examine the pattern of the gastric secretory response to insulin in dogs, to elucidate the mechanism of the response, and in particular, to consider the role in it of the adrenal glands.

\section{THE PATTERN OF THE RESPONSE TO INSULIN INJECTION}

METHODS Five dogs were equipped with innervated gastric pouches of the Pavlov type and four dogs with the Heidenhain type of denervated pouches. Pouch drainage was achieved with silver cannulae. 
Soluble insulin (B.W.) was injected intravenously in a dose of either 1 unit or 1.5 units per kilogram body weight. Occasionally as much as 2.5 units per $\mathrm{kg}$. was found necessary to induce hypoglycaemia. The gastric secretory response to insulin, as well as the ability to induce hypoglycaemia, varies from dog to dog and a satisfactory standard dose has to be sought for each animal. Blood was withdrawn at intervals by venepuncture and the level of plasma glucose determined by a modification of the method of Nelson and Somogyi (1953).

A cephalic phase of gastric secretion was stimulated by tease feeding. For a period of 15 minutes fresh minced beef and liver were fried in front of the fasting animal during which time the food was used deliberately to tease and excite.

PROCEDURE The stand-trained dogs were fasted for 18 hours before each experiment and the gastric secretion was collected continuously by gravity drainage from the cannula. The collecting tube was changed every 30 minutes and the volume of the contents measured and titrated against N10 sodium hydroxide, with Töpfer's reagent as indicator. When basal secretion was minimal the stomach was stimulated by either the intravenous injection of insulin or by tease feeding. The subsequent secretion from the pouches was collected for eight hours.

Control experiments included the measurement of the eight-hour pouch responses to prolonged fasting, to a single mixed meal, and to the injection of a 'maximal' subcutaneous dose of histamine acid phosphate. In the first group of control experiments the standard conditions were followed, the dogs remaining in the stands for eight hours, and an intravenous injection of $1 \mathrm{ml}$. of normal saline was given when the basal output was minimal and steady. Three of these control experiments were carried out at night to obviate any possibility of variations in the gastric secretory response being due to diurnal rhythms or conditioning to meal times.

The control mixed meal consisted of a standard quantity of meat and biscuit and was fed to the dogs when the fasting output was minimal and steady.

The control dose of histamine acid phosphate varied from 0.4 to $0.8 \mathrm{mg}$. per $\mathrm{kg}$. in individual dogs and was covered by a prior injection of $100 \mathrm{mg}$. of mepyramine maleate to diminish undesirable side-effects.

\section{RESULTS}

RESPONSE TO INSULIN OF INNERVATED POUCHES Thirty-one experiments were made on five dogs. In all experiments gastric secretion began to increase within 30 to 45 minutes after the injection of insulin.

In two experiments the output remained fairly constant over the whole of the eight-hour period of observation.

In 26 experiments the pattern of the secretory response fell into two distinct phases, the first phase beginning 30 to 45 minutes after insulin injection and proceeding for a mean duration of two hours (range one to three hours). Thereafter secretion diminished but rose again between four and six hours from the time of injection of insulin (mean 4 hours 45 minutes) to constitute the second phase of the response, the mean duration of which was one hour fifteen minutes (range one to three hours) (Fig. 1).

In three experiments only the first phase of secretion appeared.

The total output of hydrochloric acid was greater in the second phase than in the first in nine out of the 26 experiments.

THE RESPONSE TO INSULIN OF DENERVATED POUCHES Nineteen experiments were made on four dogs. In 17, augmentation of the basal secretion did not appear during the first three hours after the injection of insulin. In three experiments a rise in the secretory output failed to appear throughout the eight-hour observation period. In two experiments a small but definite secretory response occurred within an hour of insulin injection and persisted for two hours.

In 16 experiments a prominent acid secretory response began three and a half hours after the injection of insulin with a peak between four and a half and five hours and a mean duration of two hours forty minutes (range one to three hours 30 minutes) (Fig. 2).

The character of these delayed responses appears similar to that of the second phase observed in the stimulation of innervated pouches.

EFFECT ON SECRETORY RESPONSE TO INSULIN OF SUBSEQUENT DENERVATION OF AN INNERVATED POUCH After three standard experiments to determine the response of a Pavlov pouch to insulin the dog was further operated upon and the pouch converted to the Heidenhain type by simple excision of its connexions to the main stomach. Subsequently, the secretory response to insulin was determined on four occasions.

The effect of conversion of the pouch was to abolish the first phase of the secretory response to insulin (Fig. 3). The pattern of the delayed response was unchanged.

The decrease in the absolute values of the response after denervation is mainly the result of the loss of vagal 'tone' to the parietal cells. The reduction after denervation of the total response by two-thirds is in accord with our experience of the effect of vagotomy upon insulin- and histamine-stimulated gastric secretion in humans (unpublished data).

RELATION OF PLASMA GLUCOSE LEVELS TO BIPHASIC GASTRIC SECRETORY RESPONSE TO INSULIN In 10 

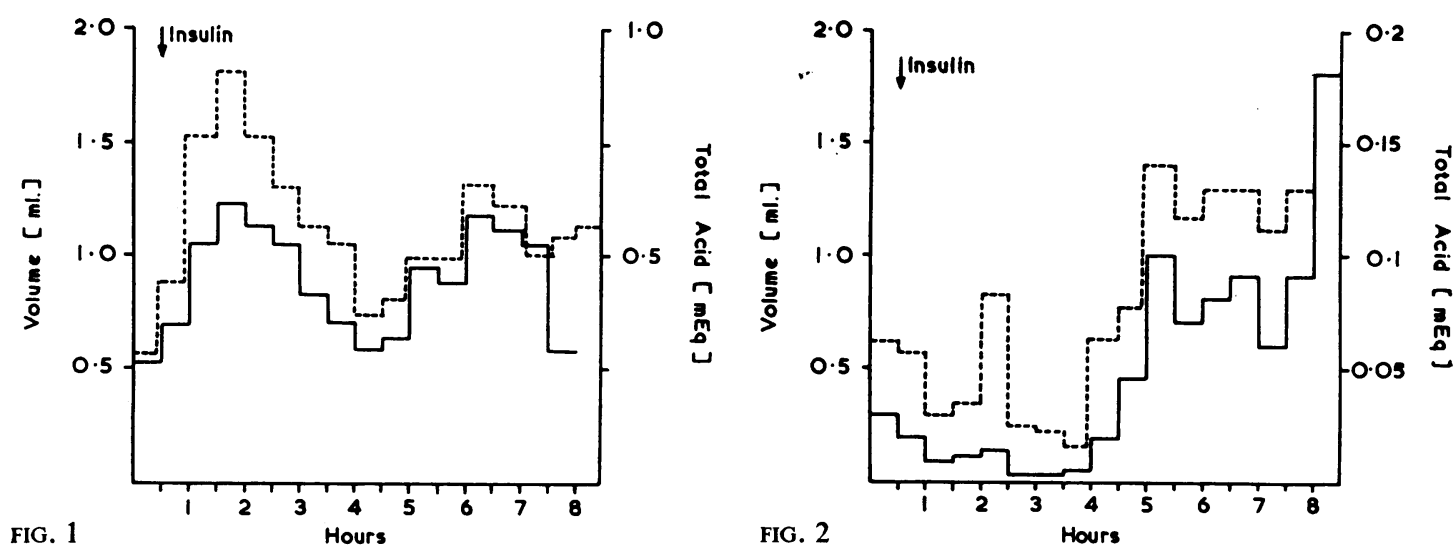

FIG. 1. The response to insulin of innervated gastric pouches (means of 31 experiments).

\begin{tabular}{lllllllllllllllll} 
Half-hour periods & Basal & 1 & 2 & 3 & 4 & 5 & 6 & 7 & 8 & 9 & 10 & 11 & 12 & 13 & 14 & 15 \\
\hline Volume in ml. & $\mathbf{0 . 5 6}$ & $\mathbf{0 . 8 7}$ & 1.53 & 1.80 & 1.53 & 1.31 & 1.12 & $\mathbf{1} .05$ & $\mathbf{0} .73$ & $\mathbf{0 . 8 0}$ & $\mathbf{0 . 9 8}$ & $\mathbf{0} .98$ & 1.31 & 1.21 & $\mathbf{0 . 9 9}$ & 1.08 \\
Total output in mEq. HCl & $\mathbf{0 . 2 6}$ & $\mathbf{0 . 3 4}$ & $\mathbf{0 . 5 2}$ & $\mathbf{0 . 6 1}$ & $\mathbf{0 . 5 7}$ & $\mathbf{0 . 5 2}$ & $\mathbf{0 . 4 1}$ & $\mathbf{0 . 3 4}$ & $\mathbf{0 . 2 8}$ & $\mathbf{0 . 3 0}$ & $\mathbf{0 . 4 7}$ & $\mathbf{0 . 4 3}$ & $\mathbf{0 . 5 9}$ & $\mathbf{0 . 5 6}$ & $\mathbf{0 . 5 2}$ & $\mathbf{0 . 3 8}$
\end{tabular}

The mean duration of the period separating the two phases is two hours eleven minutes.

FIG. 2. The response to insulin of denervated gastric pouches (means of 19 experiments).

\begin{tabular}{lllllllllllllllll} 
Half-hour periods & Basal & 1 & 2 & 3 & 4 & 5 & 6 & 7 & 8 & 9 & 10 & 11 & 12 & 13 & 14 & 15 \\
\hline Volume in ml. & 0.61 & 0.57 & 0.30 & 0.35 & 0.82 & 0.260 & 0.230 & 0.17 & 0.63 & 0.77 & 0.14 & 1.20 & 1.30 & 1.30 & $1 \cdot 10$ & 1.30 \\
Total output in mEq. $\mathrm{HCl}$ & 0.03 & 0.02 & 0.01 & 0.01 & 0.02 & 0.004 & 0.005 & 0.02 & 0.04 & 0.10 & 0.70 & 0.80 & 0.90 & 0.06 & 0.90 & 0.18
\end{tabular}
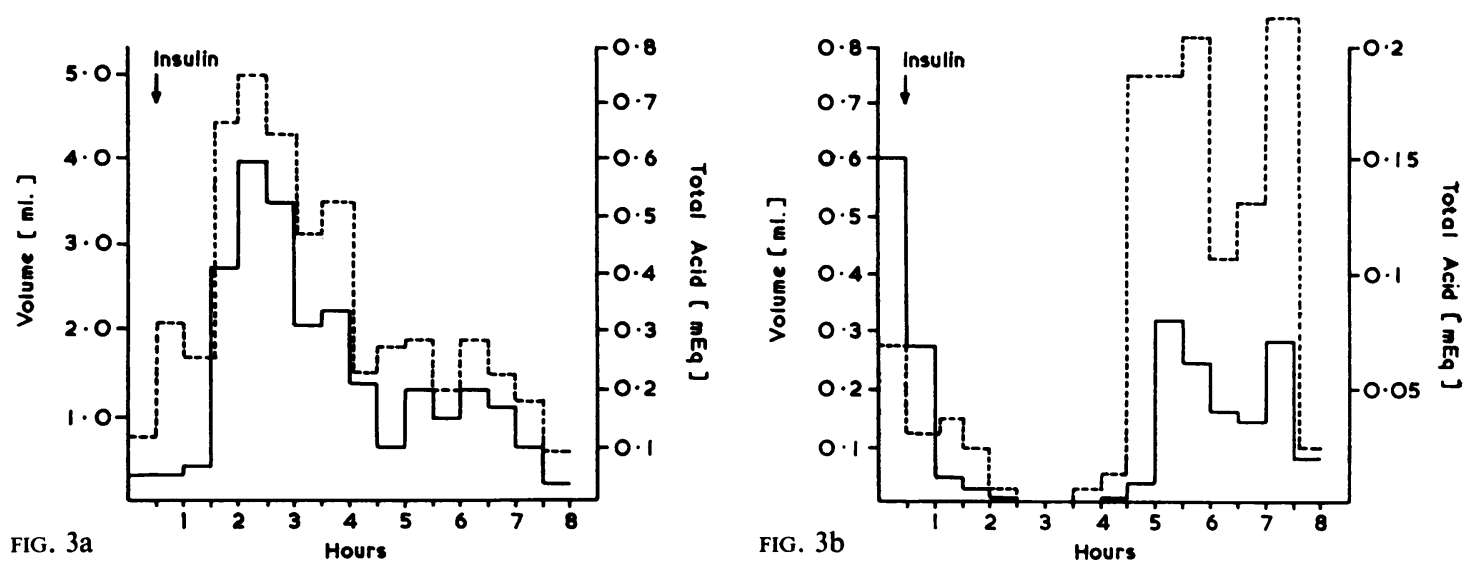

FIG. 3. The effect of denervation of an innervated pouch upon the secretory response to insulin.

$\begin{array}{llllllllllllllllll}\text { Half-hour periods } & \text { Basal } & 1 & 2 & 3 & 4 & 5 & 6 & 7 & 8 & 9 & 10 & 11 & 12 & 13 & 14 & 15\end{array}$ Means of three experiments before conversion

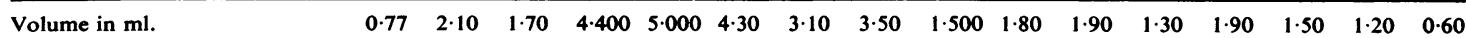

$\begin{array}{lllllllllllllllll}\text { Total output in mEq. } \mathrm{HCl} & 0.04 & 0.04 & 0.06 & 0.410 & 0.590 & 0.52 & 0.31 & 0.33 & 0.210 & 0.10 & 0.20 & 0.15 & 0.20 & 0.17 & 0.10 & 0.03\end{array}$

Means of four experiments after conversion to a denervated pouch

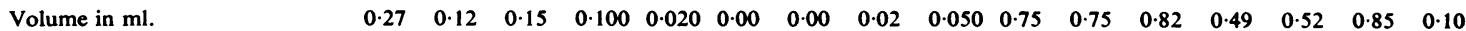

$\begin{array}{lllllllllllllllll}\text { Total output in mEq. HCl } & 0.15 & 0.07 & 0.01 & 0.005 & 0.002 & 0.00 & 0.00 & 0.00 & 0.002 & 0.01 & 0.08 & 0.06 & 0.04 & 0.04 & 0.07 & 0.02\end{array}$

The scales have been adjusted to demonstrate more clearly the pattern of the response.

$$
\text { Continuous line }=\text { total acid } \quad \text { Broken line }=\text { volume }
$$


experiments on four dogs blood was withdrawn at intervals throughout the eight hours and the level of glucose in the plasma determined. Blood samples were removed every 15 minutes for the first one and a half hours, hourly for the subsequent three hours, and every 30 minutes for the remainder of the experiment. The peak point of hypoglycaemia appeared at approximately 45 minutes from the time of injection of insulin at which time the first phase of the gastric secretory response had begun from innervated pouches. By the end of this phase of secretion the plasma glucose levels were returning to normal and at the commencement of the second secretory phase had reached the basal level or lay above it. Thereafter, no significant variation appeared in the level of plasma glucose.

The second phase of the gastric secretory response began at a mean time of three hours from the point of maximal hypoglycaemia. No differences occurred in the plasma glucose levels after insulin between dogs with denervated and those with innervated pouches and satisfactory hypoglycaemia was obtained in all experiments whether or not, depending on the type of pouch, a first phase of gastric secretion appeared (Table I, Fig. 4).

\section{TABLE I}

VARIATION IN LEVELS OF PLASMA GLUCOSE AFTER INJECTION OF INSULIN ${ }^{1}$

Before After Insulin

Insulin

\begin{tabular}{|c|c|c|c|c|c|c|}
\hline $\begin{array}{l}\text { Time (hr.) } \\
\text { Plasma glucose } \\
\text { (mg. \%) }\end{array}$ & $\begin{array}{l}0 \\
67.9\end{array}$ & $\begin{array}{l}34 \cdot 3 \\
34 \cdot 3\end{array}$ & 38.6 & $\begin{array}{l}2 \frac{1}{2} \\
51 \cdot 0\end{array}$ & $\begin{array}{l}4 \frac{1}{2} \\
71 \cdot 4\end{array}$ & $5 \frac{1}{2}$ \\
\hline
\end{tabular}

${ }^{1}$ The means of 10 experiments. A representative experiment is illustrated (Figure 4).

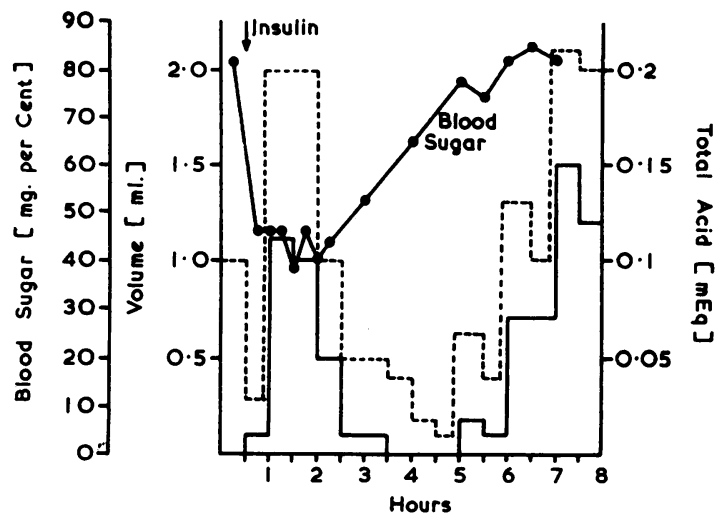

FIG. 4. The relation of the blood sugar levels in a dog with an innervated pouch to the gastric secretory response to insulin in a representative experiment (see Table I).

Continuous line $=$ total acid

Broken line = volume

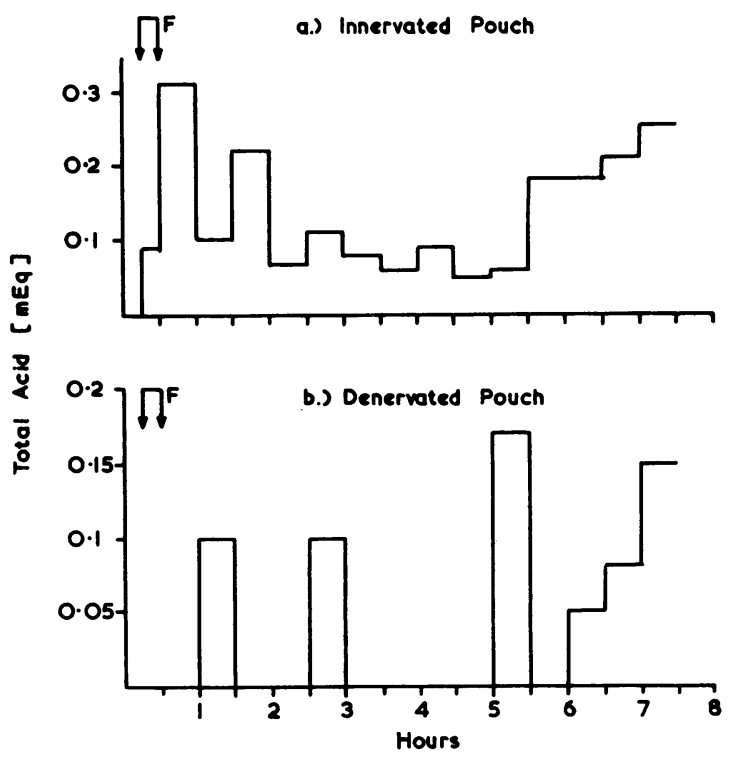

FIG. 5. Two experiments illustrating the appearance of biphasic responses to tease feeding (a) in an innervated pouch, and (b) in a denervated pouch. The scales have been altered to allow better appreciation of the pattern of the response.

$F=15$ min., frying liver and mince.

SECRETORY RESPONSES OF GASTRIC POUCHES TO CENTRAL STIMULATION BY TEASE FEEDING Eleven experiments were made on five dogs, two with innervated and three with denervated pouches.

In four of the five experiments with innervated pouches the secretory response showed a biphasic response.

In two of the six experiments with denervated pouches similar double phasing appeared, in two others only a delayed response was obtained, and of the remaining experiments one showed no response at all and the other a continuous secretion throughout.

When present, the first phase appeared immediately, lasted for one to two hours and was followed by a period of basal response continuing thereafter for two to three hours. The second phase of augmented secretory activity commenced four to four and a half hours after the tease feeding and lasted one and a half to two hours (Fig. 5).

\section{CONTROL EXPERIMENTS}

RESPONSE OF GASTRIC POUCHES TO PROLONGED FASTING AND INJECTION OF INTRAVENOUS SALINE Eleven experiments were made on four dogs, three 

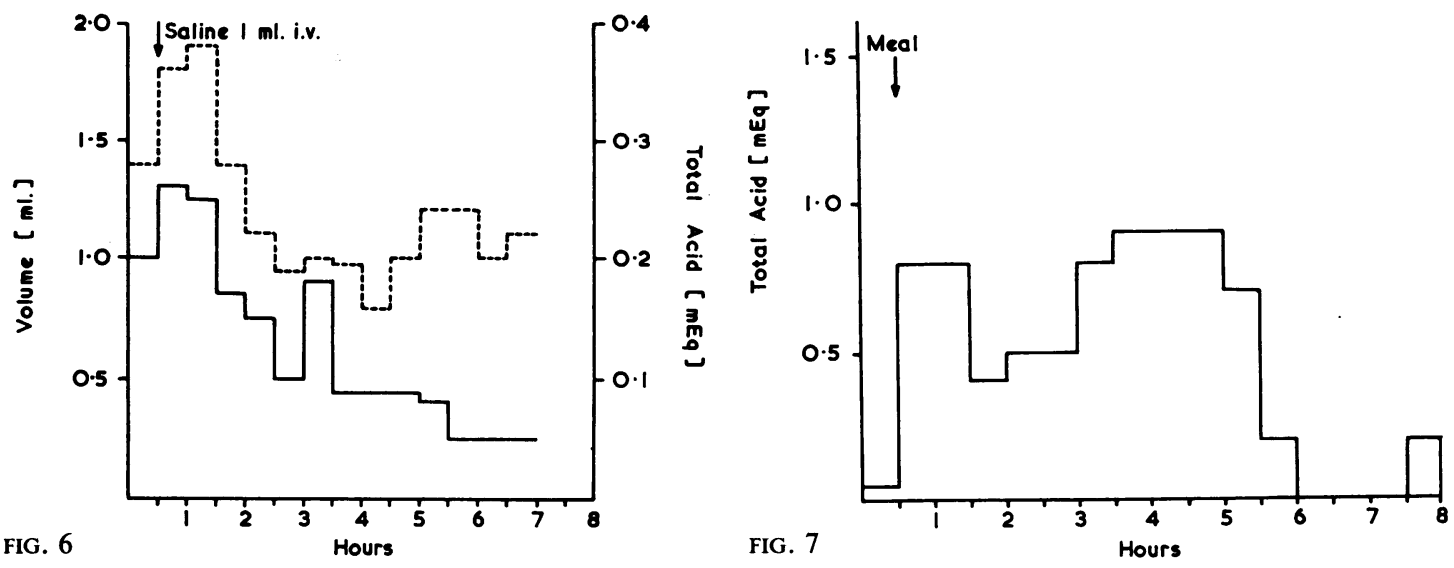

FIG. 6. The secretory response of gastric pouches (both pouch types) to prolonged fasting and a single intravenous injection of $1 \mathrm{ml}$. saline in control experiments (the means of 11 experiments of volume and total acid output for each half-hour period). Continuous line = total acid Broken line = volume

FIG. 7. The secretory response of a denervated pouch to the feeding of a mixed meal showing phasic fluctuation.

with innervated pouches and one with a denervated pouch (Fig. 6).

In none of the experiments was any significant fluctuation in the basal acid output observed. There was no rise in secretion corresponding to the delayed response to insulin hypoglycaemia.

Fluctuation in the volume of secretion was observed in most experiments but this was due to varying flows of mucus and did not significantly affect the acid output.

RESPONSE OF GASTRIC POUCHES TO FEEDING OF A MIXED MEAL Three experiments were made, two on innervated pouch dogs and one on a denervated pouch dog.

A brisk initial response was obtained in all experiments but the secretion steadily declined over the eight hours in two of the experiments without any delayed period of increased secretion. The Heidenhain pouch, however, appeared to show a phase of augmented response between three and four and a half hours after the feed similar to that observed in the tease feeding experiments (Fig. 7).

RESPONSE OF GASTRIC POUCHES TO A SINGLE 'MAXIMAL' SUBCUTANEOUS INJECTION OF HISTAMINE ACID PHOSPHATE Three experiments were made on two dogs with innervated pouches.

Secretion appeared 30 minutes after the injection of histamine, was maximal over the next hour, and dwindled to negligible proportions over a further hour. Thereafter for a further four and a half hours no significant secretion appeared (Fig. 8).

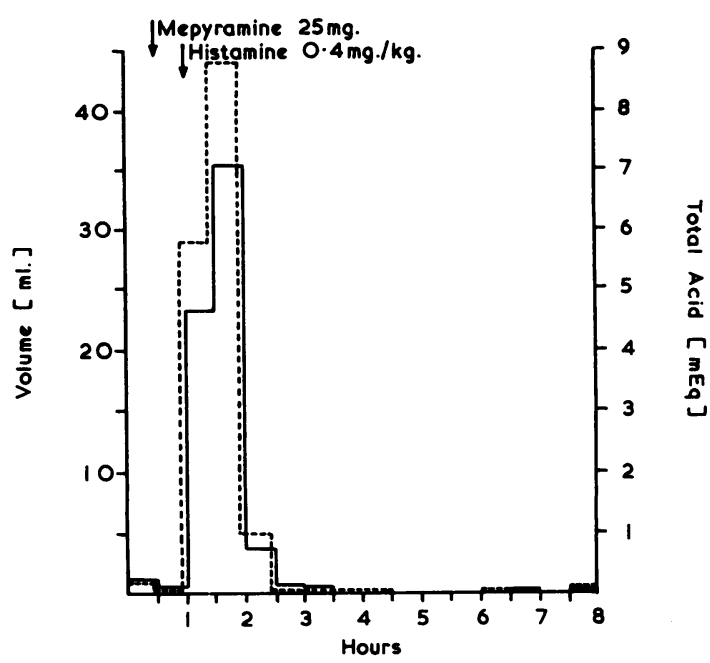

FIG. 8. The response of an innervated gastric pouch to a single 'maximal' dose of histamine followed for eight hours. Continuous line = total acid

Broken line $=$ volume

\section{DISCUSSION}

The gastric secretory response to the injection of insulin has been under examination since Collazo and Dobreff (1924) claimed that insulin depressed the secretory responses of Pavlov pouches in dogs. Subsequently, the intravenous administration of insulin was shown to promote gastric secretion with a maximal response one hour after the injection 
(Simici, Popesco, and Diculesco, 1927). The first clinical test of gastric secretion using insulin stimulation was developed by Roholm (1930) who demonstrated that there was no true correlation between the degree of hypoglycaemia or the absolute fall in the blood sugar level and the gastric secretory response. This occurred whenever the blood sugar level fell below $50 \mathrm{mg} . \%$. Roholm's two-hour insulin test is still the standard pattern used in clinical practice. All the numerous studies of the phenomenon published between 1930 and 1953 are confined to the secretory response occurring within two hours of the administration of insulin. Yet in Roholm's original data it can be seen that in seven of the 10 control experiments unstimulated secretion was maintained and rising after two hours, at which time the response following insulin was rapidly falling below the level of unstimulated secretion, suggesting that the insulin injection had initiated influences on gastric secretion, aspects of which were prolonged beyond two hours.

The studies of Porter et al. (1953) and of French et al. (1953) were the first in which the secretory response to neural and insulin stimulation was examined over more than a few hours. Unfortunately, only hydrogen ion concentration was measured electrometrically, and neither volume nor output of acid was recorded.

Shay and Sun (1954) attempted a study of insulin-induced gastric secretion in man and claimed to demonstrate the same phenomenon of a vagal and an 'adrenal' phase, as had been shown in monkeys, and with the same relationship between responses and stimuli. However, the data provided in their report are inadequate and discrepancy appears between the published graphs and the authors' interpretations and conclusions.

Whereas until the last decade the sole mediators of the gastric phase of secretion were held to be chemical and mechanical (Babkin, 1950) and the phases of gastric secretion were considered to be sharply divisible into cephalic, gastric, and intestinal, numerous studies (Uvnäs, 1942; Dragstedt, Oberhelman, Woodward, and Smith, 1952; Burstall and Schofield, 1953; Oberhelman, Rigler, and Dragstedt, 1957; Thein and Schofield, 1959a, and b; Gregory and Tracy, 1960) have demonstrated the interdependence of the mechanisms-neural, mechanical, and humoral-and the overlapping of the phases of secretion. Hence fluctuation in secretory response from whatever mode of stimulus might represent variation in the timing of release and action of the different mechanisms involved.
Furthermore, in any gastric acid secretory activity important influences on the characters of the response are the acid-inhibiting mechanisms of the antrum and duodenum (Woodward, Lyon, Landor, and Dragstedt, 1954; Sircus, 1958). Thus the appearance of phasing in a gastric secretory response could represent either the operation of different excitatory mechanisms at different times, or the temporary depression of an excitatory mechanism by an inhibitory one, or to an interaction of both types.

This section of the present study demonstrates that the variation in the secretory response to insulin is not due to ordinary diurnal variations in relation to hunger or feeding times or other biological rhythms. It is shown that the phasing phenomenon is peculiar to gastric secretory responses in which the stimulus involves at least in part the autonomic nervous system activated either by psychic stimulation or by hypoglycaemia. Nor does it appear as a result of the direct stimulation of parietal cells with a massive dose of histamine, or with the passage of acid per se into the antrum and small intestine.

Two prominent phases of gastric secretion appear in response to insulin administration and the first of these is dependent upon intact innervation of the secreting mucosa of the body of the stomach. The second phase is not so dependent and appears therefore to represent the activity of a humoral mechanism. Likewise the secretion by the pouch in the second phase is not dependent upon the appearance of the first phase, so that the second phase must represent either secretion initiated after a delay of hours from the application of the primary stimulus, or the release from a period of inhibition of part of a continued response to the initial stimulus. The failure of a single stimulus by histamine to promote a delayed phase is in sharp contrast to the biphasic or continuous response to wholly neural stimulated secretion or to meals. The latter two differ from the histamine mechanism in the involvement of the antrum in the total process of stimulating the production of acid. The temporal characters of the response showed no essential differences when they occurred in innervated or denervated pouches or whether the trigger was a peak of hypoglycaemia or a period of tease feeding. The first of the phases correlated with the onset of hypoglycaemia but plasma glucose levels are back to normal before the second phase appears so that the phasing is not a reflection of the effects of fluctuation in the blood sugar values after insulin. 